\title{
Sensitivity of the gamma-ray method for strong primordial magnetic fields
}

\author{
A.Korochkin, ${ }^{a, b, c, *}$ O.Kalashev, ${ }^{a, c, d}$ A.Neronov $^{b, e}$ and D.Semikoz ${ }^{b}$ \\ ${ }^{a}$ INR RAS, \\ 60 October anniversary prosp. 7a, Moscow, Russia \\ ${ }^{b}$ Université de Paris, CNRS, Astroparticule et Cosmologie, \\ F-75013 Paris, France \\ ${ }^{c}$ Novosibirsk State University, \\ Pirogova 2, Novosibirsk, 630090 Russia \\ ${ }^{d}$ Moscow Institute for Physics and Technology, \\ 9 Institutskiy per., Dolgoprudny, Moscow Region, 141701 Russia \\ ${ }^{e}$ Astronomy Department, University of Geneva, \\ Ch. d'Ecogia 16, 1290, Versoix, Switzerland \\ E-mail: aa.korochkin@physics.msu.ru
}

We study the sensitivity reach of the gamma-ray method for measurement of relatively strong cosmological magnetic field with strength in the $1-10 \mathrm{pG}$ range using deep exposure of the nearest hard spectrum blazar Mrk 501 with CTA telescopes. We show that the gamma-ray measurement technique can sense the primordial magnetic field with a strength of up to $10^{-11} \mathrm{G}$. Combination of the cosmic microwave background and gamma-ray constraints can thus sense the full range of possible cosmological magnetic fields to confirm or rule out their relevance to the problem of the origin of cosmic magnetic fields, as well as their influence on recombination and reionization epochs.

$37^{\text {th }}$ International Cosmic Ray Conference (ICRC 2021)

July 12th - 23rd, 2021

Online - Berlin, Germany

\footnotetext{
*Presenter
} 


\section{Introduction}

Magnetic fields are present in almost every observable astronomical object. Yet, their existence and role in the early universe is uncertain. A combination of $\gamma$-ray lower bounds [1,2] and radio and CMB upper bounds [3, 4] on Intergalactic Magnetic Field (IGMF) provides an evidence for existence of fields with the strength $10^{-16} \mathrm{G}<B<10^{-9} \mathrm{G}$ in the intergalactic medium (see Fig. 1) $[5,6]$. However, primordial nature of these fields is yet to be established.

[7] have shown that account of the magnetic field driven turbulence on plasma at the epoch of recombination can relax the $4.4 \sigma$ tension between estimates of the Hubble parameter from the Cosmic Microwave Background (CMB) measurement [8] and present-day universe measurements using supernovae type Ia [9] and based on gravitationally lensed systems [10]. The resulting magnetic field strength $B \propto 10^{-11} \mathrm{G}$.

Another indication of existence of cosmological magnetic fields can be derived from measurements of redshift dependent absorption by the $21 \mathrm{~cm}$ line of neutral hydrogen at redshifts about $z \sim 10$. EDGES experiment has recently reported an excess opacity of the Universe in the redshift range $15<z<20$ [11]. The possible explanation of the EDGES data requires magnetic field in the range $5 \times 10^{-13} \mathrm{G}<B<6 \times 10^{-12} \mathrm{G}$. [12].

As shown in $[13,15]$ presence of helical magnetic field at the epoch of Electroweak phase transition can enable explanation of the baryon asymmetry of the universe within the Standard Model of particle physics. This requires the range of magnetic field strength $10^{-14} \mathrm{G}<B<10^{-12} \mathrm{G}$.

Combination of these observational hints for existence of cosmological magnetic field defines an order-of-magnitude wide "sweet spot" around $B \sim 10^{-12} \mathrm{G}$ in which the field estimates from multiple effects intersect. We demonstrate that even though the field is at the upper sensitivity end of the $\gamma$-ray technique, its detection should still be possible with a deep exposure of the nearest blazars with CTA [16].

\section{Analytical estimates}

Fields with the strength in the range $B \sim 10^{-12} \mathrm{G}$ are at the upper end of the sensitivity reach of the $\gamma$-ray measurement method [5]. They are strong enough to deflect trajectories of electrons with energies in 10-100 TeV range. This implies that the highest energy $\gamma$-ray signal accessible to telescopes should be used for the signal measurements. In this situation it is not clear if the small angle deflection approximation previously used for the sensitivity estimates used by [5] is valid. We re-assess the analytical estimates in this high-energy / strong field regime below.

We consider secondary emission induced by interactions of primary $\gamma$-rays with energies $E_{\gamma 0}$. The mean free path of these $\gamma$-rays through the EBL is

$$
\lambda_{\gamma 0} \simeq 2.5\left[\frac{E_{\gamma 0}}{100 \mathrm{TeV}}\right]^{-1.6} \mathrm{Mpc}
$$

For the analytical estimates we assume that each primary $\gamma$-ray produces electron and positron with energies $E_{e}=E_{\gamma 0} / 2$. Such electrons produce inverse Compton emission at the energy

$$
E_{\gamma} \simeq 8\left[\frac{E_{e}}{50 \mathrm{TeV}}\right]^{2} \mathrm{TeV}
$$




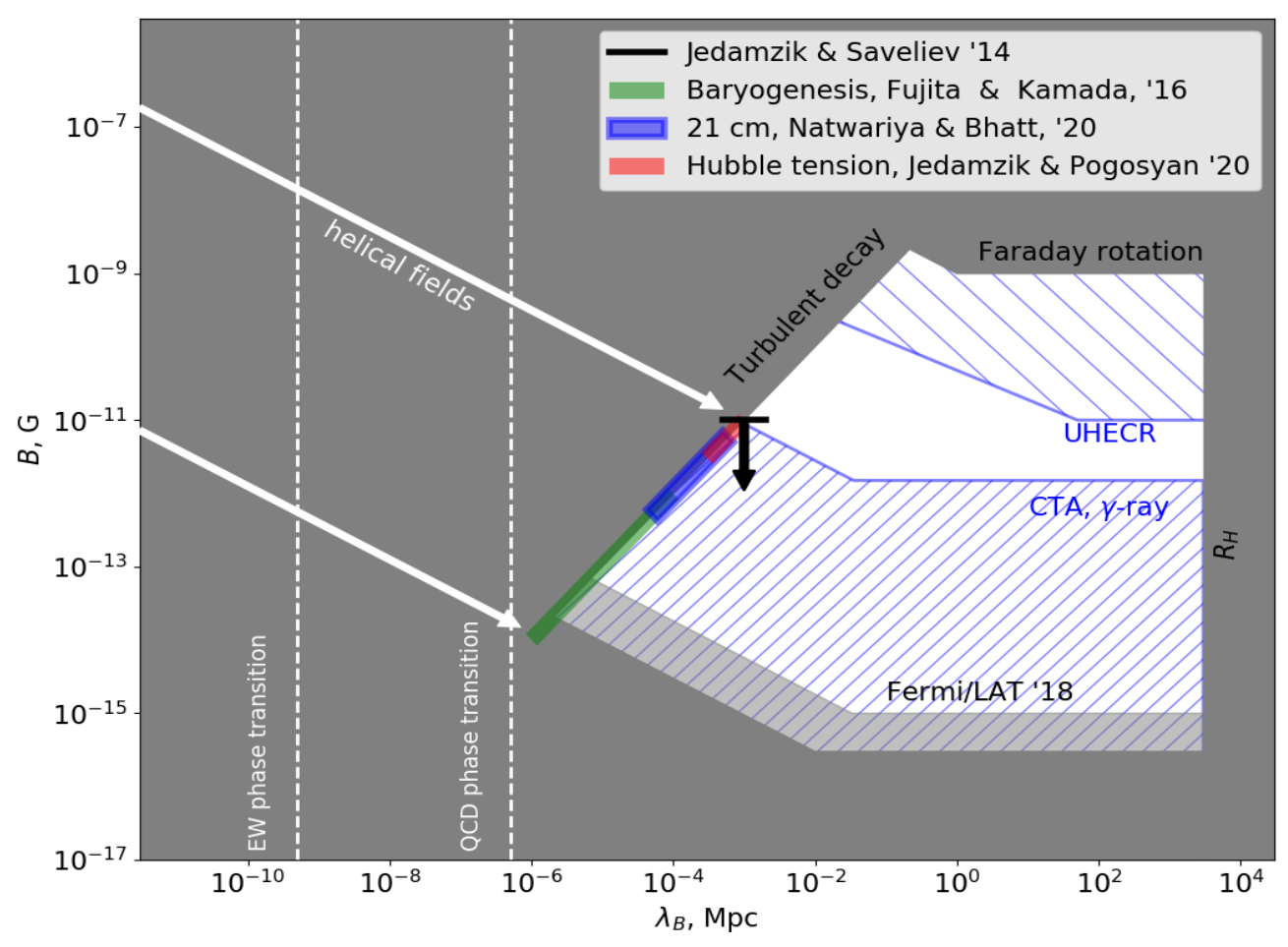

Figure 1: Known constraints on IGMF [5, 6] and hints of existence of cosmological magnetic field from CMB [7], $21 \mathrm{~cm}$ line [12] and baryogenesis [13], compared to the sensitivity of different detection techniques. Black upper bound is from the analysis of CMB signal by [14].

Accumulation of such small deflections on the electron cooling distance scale $D_{e}$ results in the overall deflection

$$
\Delta=\sqrt{\frac{D_{e}}{\lambda_{B}}} \delta \simeq 0.2\left[\frac{E_{e}}{50 \mathrm{TeV}}\right]^{-3 / 2}\left[\frac{B}{10^{-11} \mathrm{G}}\right]^{3 / 2} \simeq 0.2\left[\frac{E_{\gamma}}{8 \mathrm{TeV}}\right]^{-3 / 4}\left[\frac{B}{10^{-11} \mathrm{G}}\right]^{3 / 2}
$$

where $R_{L}$ is the electron's gyroradius and we used that the correlation length $\lambda_{B}$ of cosmological magnetic fields scales with the strength as [17] $B \sim 10^{-11}\left[\lambda_{B} / 1 \mathrm{kpc}\right] \mathrm{G}$

If the field strength is $B \sim 10^{-11} \mathrm{G}$, the opening angle of the secondary emission cone at $8 \mathrm{TeV}$ can be as large as opening angle of the AGN jets. The secondary emission flux within the cone gets suppressed as

$$
\frac{F_{\text {ext }}}{F_{\gamma 0}}=\frac{\Theta_{j e t}^{2}}{\Delta^{2}} \simeq 1\left[\frac{E_{\gamma}}{8 \mathrm{TeV}}\right]^{3 / 2}\left[\frac{B}{10^{-11} \mathrm{G}}\right]^{-3}\left[\frac{\Theta_{j e t}}{10^{\circ}}\right]^{2}, \quad B \gtrsim 10^{-11} \mathrm{G}
$$

where we have assumed $\Theta_{\text {jet }} \sim 10^{\circ} \simeq 0.2$. Such flux suppression occurs below the energy $E_{\text {crit }}$ at which $\Delta=\Theta_{\text {jet }}$.

If $B<10^{-11} \mathrm{G}$, the deflection angle $\Delta$ is smaller than the opening angle of the jet and extended emission is still observable toward $10 \mathrm{TeV}$ energy. The maximal possible angular size of the 

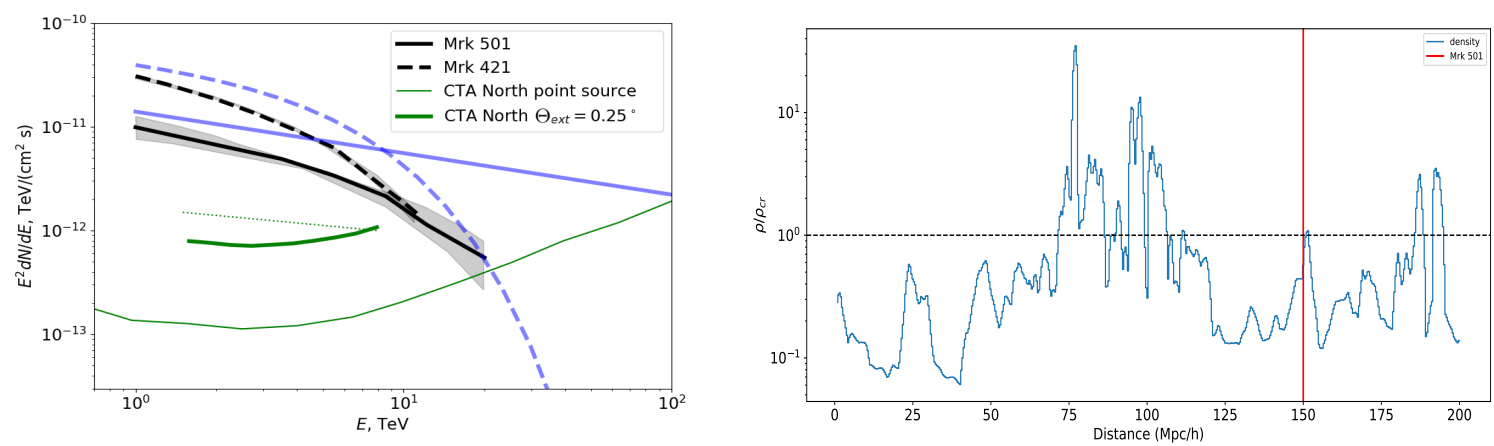

Figure 2: Left: Comparison of high-energy ends of the spectra of Mrk 421 and Mrk 501 [18]. Black curves are observed, blue curves are the intrinsic spectra of the sources. Green line shows CTA North point source sensitivity. Green dotted line shows an analytical estimate of the secondary $\gamma$-ray flux from Mrk 501 in 1-10 energy range assuming no influence of IGMF. Right: Dark matter density profile along the line of sight towards Mrk 501 based on constrained simulations of the LSS [? ].

extended emission is determined by the transverse size of the jet at the distance $\lambda_{\gamma 0}$ and corresponds to the angular size

$$
\Theta_{\text {ext }, \text { max }}=\frac{R_{\text {ext }}}{D} \simeq 0.24^{\circ}\left[\frac{\Theta_{j e t}}{10^{\circ}}\right]\left[\frac{E_{\gamma}}{8 \mathrm{TeV}}\right]^{-0.8}\left[\frac{D}{120 \mathrm{Mpc}}\right]^{-1}
$$

where we have used the distance to Mrk 421 and Mrk 501 for the numerical estimate. The time delay of the extended signal can be estimated as $T_{\text {ext, } \text { max }}=D \Theta_{\text {ext, } \text { max }}^{2} / c \propto 10 \mathrm{kyr}$ and imposes a requirement on the duty cycle of the source for which the extended emission is detectable: the source should have been active over the last $10 \mathrm{kyr}$.

\section{Selection of best target for the search of strong IGMF}

Probe of the strongest fields $B \lesssim 10^{-11} \mathrm{G}$ requires

(a) large primary point source power in the $100 \mathrm{TeV}$ energy range;

(b) detectability of extended emission in multi-TeV energy range;

(c) favorable local environment around the source.

Below we present arguments that at least one source, Mrk 501, fulfils these three conditions and can be used for the probe of strong IGMF of cosmological origin.

The highest energy photons detected from blazars are those from the two nearest BL Lacs, Mrk 421 and Mrk 501. Fig. 2 shows the spectral energy distribution of these sources measured by HAWC [18]. One can see that Mrk 421 intrinsic luminosity is most probably strongly suppressed at $100 \mathrm{TeV}$. To the contrary, Mrk 501 has harder spectrum which does not show any signature of high-energy cut-off. In the view of this fact, we consider Mrk 501 as a more promising candidate for the search of the strongest IGMF and the following calculations are limited to this source. We assume that its intrinsic spectrum extends up to $100 \mathrm{TeV}$, as shown in Fig. 2. 


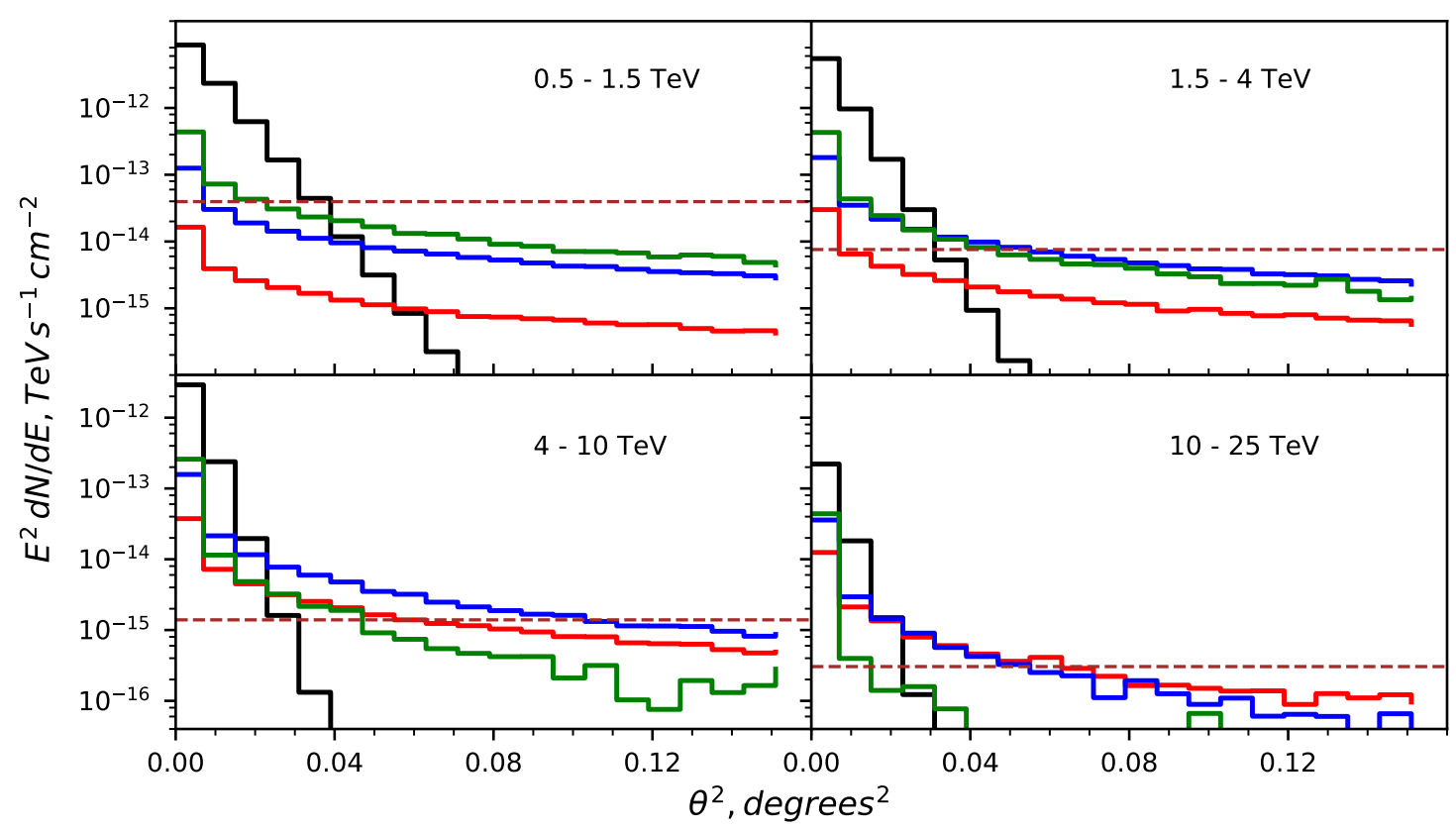

Figure 3: Angular distribution of primary and secondary photons in different energy ranges. Black histograms show the primary point source signal, green, blue and red histograms show the extended emission calculated for different magnetic field strengths: $10^{-12} \mathrm{G}, 3 \times 10^{-12} \mathrm{G}, 10^{-11} \mathrm{G}$. Horizontal dashed line shows the level of residual cosmic ray electron background measured by HESS [23].

As was shown in [19] large magnetized bubbles blown by the source host galaxy can effectively remove secondary gamma-ray flux. Strong $\left(B>10^{-12} \mathrm{G}\right)$ magnetic fields in the bubbles sufficiently deflect charged particles created in the bubbles and suppress their secondary gamma-ray flux at the detector all across its energy range. This changes the overall normalization of the secondary emission, without changing its shape. The average flux suppression can reach $20 \%-50 \%$ for hypothetical sources with hard intrinsic spectra extending up to $E_{\mathrm{cut}}=100 \mathrm{TeV}$ if the size of the bubble is larger than several Mpc. Therefore, local environment of the source should be taken into account in the analysis of its secondary flux.

To explore environmental effects around Mrk 501, we rely on constrained cosmological simulations derived using the BORG inference method [20-22]. From Fig.2 one can see that the source is not found in a node of the LSS and hence does not appear to be close to a rich galaxy cluster but rather in an underdense region with the average density $\rho$ below the critical density of the universe. This means that the IGMF around the source should not be amplified in the course of structure formation.

\section{Numerical modelling}

In this section we support this qualitative argument with numerical modelling of the extended source signal around Mrk 501, which is the brightest blazar in the $10 \mathrm{TeV}$ sky. For this purpose we use the Monte-Carlo simulation code CRbeam developed in [24]. 

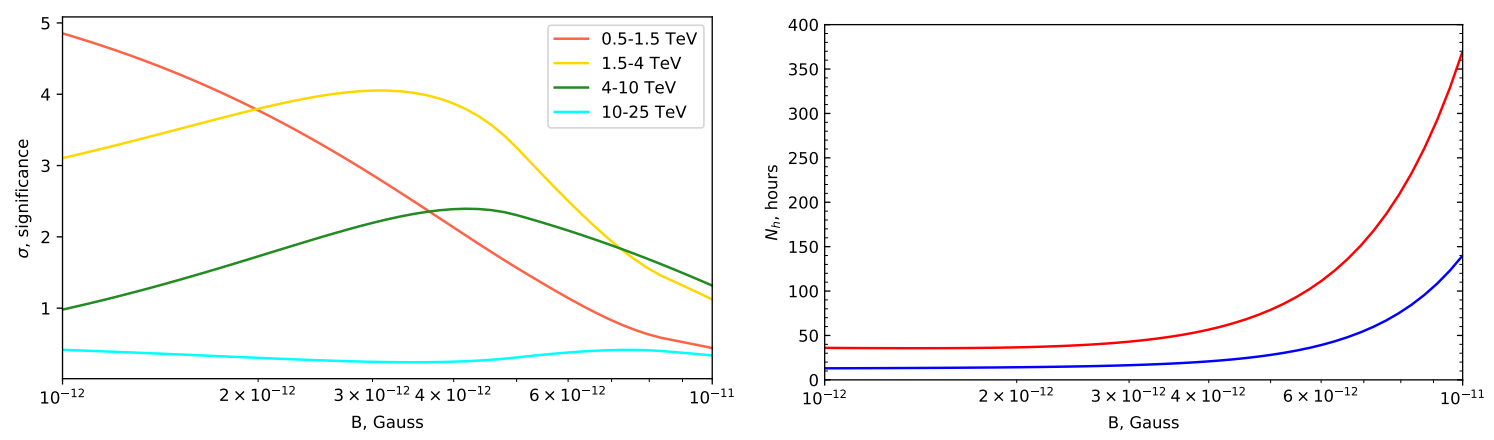

Figure 4: Left:Significance of detection of the extended emission signal in different energy ranges as a function of the assumed magnetic field strength. The assumed exposure of CTA is $T=50 \mathrm{hr}$. Right: CTA exposure time needed for $3 \sigma$ (lower curve) and $5 \sigma$ (upper curve) detection of the extended emission signal, as a function of IGMF strength.

We consider a primary $\gamma$-ray source with the powerlaw spectrum with the slope $d N / d E \propto E^{-2.4}$ extending up to $100 \mathrm{TeV}$ energy, situated at the distance $D=150 \mathrm{Mpc}$. The $\gamma$-rays are emitted into a jet with opening angle $\Theta_{j e t}=5^{\circ}$ aligned along the line of sight. We simulate propagation of primary $\gamma$-ray, taking into account pair production and inverse Compton scattering on CMB and Extragalactic Background Light (model of [25]) and deflection of the charged component in magnetic field. The magnetic field is generated with Kolmogorov power spectrum. Fig. 3 shows the result of calculation of extended emission pattern at different energies for a range of IGMF strengths.

\section{Results and discussion}

We have used the results of the Monte-Carlo modelling to investigate detectability of the extended emission signal with CTA. To do this, we have calculated the statistics of the point source signal, extended emission signal and residual cosmic ray background in each angular bin of the histograms shown in Fig. 3, for different CTA exposures. In this way we have generated mock CTA datasets. We have fitted the mock data with a model of point source plus residual cosmic ray background model, ignoring the presence of the extended source. We have then estimated the significance of detection of the extended emission in the simulated data set by calculating the level of inconsistency of the "point source + residual cosmic ray background" model with the simulated data.

The results of this analysis are shown in Fig. 4. If the IGMF strength is below $3 \times 10^{-12} \mathrm{G}$, the extended signal is detectable with significance larger than $3 \sigma$ in the energy ranges $0.5-1.5 \mathrm{TeV}$ and $1.5-4 \mathrm{TeV}$. Somewhat stronger magnetic field, $3 \times 10^{-12}<B<6 \times 10^{-12} \mathrm{G}$ is still marginally detectable through the extended emission at somewhat higher energy, up to $10 \mathrm{TeV}$. The extended emission signal associated to the $10^{-11} \mathrm{G}$ IGMF is not detectable in a $50 \mathrm{hr}$ exposure. Fig. 4 shows the exposure needed for the $3 \sigma$ evidence for and $5 \sigma$ discovery of the extended emission for different IGMF strengths. From this figure one can see that with a $150 \mathrm{hr}$ exposure, an evidence for the presence of extended emission in $10 \mathrm{TeV}$ energy range can be found even for the magnetic field with the strength $10^{-11} \mathrm{G}$. 
To summarise, we have shown that direct detection of the strong cosmological magnetic field, which is needed for the resolution of the tension between different measurements of the Hubble parameter [7] and for the explanation of the EDGES $21 \mathrm{~cm}$ line opacity [12] is possible with the $\gamma$-ray measurement technique and at least one source, Mrk 501, is suitable for this purpose.

\section{Acknowledgements}

This work has been supported by the French National Research Agency (ANR) grant ANR-19CE31-0020 and Russian Science Foundation grant 20-42-09010.

\section{References}

[1] A. Neronov and I. Vovk, Evidence for Strong Extragalactic Magnetic Fields from Fermi Observations of TeV Blazars, Science 328 (2010) 73 [1006 . 3504].

[2] M. Ackermann et al., The Search for Spatial Extension in High-latitude Sources Detected by the Fermi Large Area Telescope, Ap.J.Supp. 237 (2018) 32 [1804. 08035].

[3] P.P. Kronberg, Extragalactic magnetic fields, Reports on Progress in Physics 57 (1994) 325.

[4] Planck Collaboration, Planck intermediate results. XLII. Large-scale Galactic magnetic fields, A\&A 596 (2016) A103 [1601.00546].

[5] A. Neronov and D.V. Semikoz, Sensitivity of $\gamma$-ray telescopes for detection of magnetic fields in the intergalactic medium, Phys.Rev.D 80 (2009) 123012 [0910 . 1920].

[6] R. Durrer and A. Neronov, Cosmological magnetic fields: their generation, evolution and observation, A\&A Rv. 21 (2013) 62 [1303.7121].

[7] K. Jedamzik and L. Pogosian, Relieving the Hubble tension with primordial magnetic fields, Phys. Rev. Lett. 125 (2020) 181302 [2004 . 09487].

[8] Planck collaboration, Planck 2018 results. VI. Cosmological parameters, Astron. Astrophys. 641 (2020) A6 [1807.06209].

[9] A.G. Riess, S. Casertano, W. Yuan, L.M. Macri and D. Scolnic, Large Magellanic Cloud Cepheid Standards Provide a 1\% Foundation for the Determination of the Hubble Constant

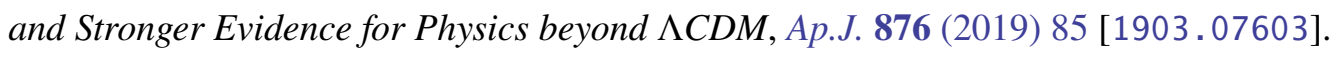

[10] K.C. Wong et al., HOLiCOW XIII. A 2.4\% measurement of $H_{0}$ from lensed quasars: $5.3 \sigma$ tension between early and late-Universe probes, MNRAS (2020) [1907.04869].

[11] J.D. Bowman, A.E.E. Rogers, R.A. Monsalve, T.J. Mozdzen and N. Mahesh, An absorption profile centred at 78 megahertz in the sky-averaged spectrum, Nature 555 (2018) 67 [1810.05912].

[12] P.K. Natwariya and J.R. Bhatt, EDGES signal in the presence of magnetic fields, Mon. Not. Roy. Astron. Soc. 497 (2020) L35 [2001 . 00194]. 
[13] T. Fujita and K. Kamada, Large-scale magnetic fields can explain the baryon asymmetry of the Universe, Phys.Rev.D 93 (2016) 083520 [1602 . 02109].

[14] K. Jedamzik and A. Saveliev, Stringent Limit on Primordial Magnetic Fields from the Cosmic Microwave Background Radiation, Phys.Rev.Lett. 123 (2019) 021301 [1804 . 06115].

[15] M. Giovannini and M.E. Shaposhnikov, Primordial hypermagnetic fields and the triangle anomaly, Phys.Rev.D 57 (1998) 2186 [hep-ph/9710234].

[16] A. Korochkin, O. Kalashev, A. Neronov and D. Semikoz, Sensitivity reach of gamma-ray measurements for strong cosmological magnetic fields, Astrophys. J. 906 (2021) 116 [2007.14331].

[17] R. Banerjee and K. Jedamzik, Evolution of cosmic magnetic fields: From the very early Universe, to recombination, to the present, Phys.Rev.D 70 (2004) 123003 [astro-ph/0410032].

[18] S. Coutiño de Leon, A.C. Alonso, D. Rosa-Gonzalez and A.L. Longinotti, Spectral analysis of the blazars Markarian 421 and Markarian 501 with the HAWC Gamma-Ray Observatory, in 36th International Cosmic Ray Conference (ICRC2019), vol. 36 of International Cosmic Ray Conference, p. 654, July, 2019 [1909. 01179].

[19] K. Bondarenko, A. Boyarsky, A. Korochkin, A. Neronov, D. Semikoz and A. Sokolenko, Account of baryonic feedback effect in the gamma-ray measurements of intergalactic magnetic fields, 2106.02690.

[20] J. Jasche and B.D. Wandelt, Bayesian physical reconstruction of initial conditions from large-scale structure surveys, Monthly Notices of the Royal Astronomical Society 432 (2013) $894[1203.3639]$.

[21] G. Lavaux and M.J. Hudson, The 2M++ galaxy redshift catalogue, Monthly Notices of the Royal Astronomical Society 416 (2011) 2840 [1105 . 6107].

[22] J. Jasche and G. Lavaux, Physical Bayesian modelling of the non-linear matter distribution: New insights into the Nearby Universe, Astronomy \& Astrophysics 625 (2019) A64 [1806. 11117].

[23] D. Kerszberg, M. Kraus and e.a. Kolitzus D., The cosmic-ray electron spectrum measured with H.E.S.S., .

[24] V. Berezinsky and O. Kalashev, High energy electromagnetic cascades in extragalactic space: physics and features, Phys. Rev. D 94 (2016) 023007 [1603.03989].

[25] R.C. Gilmore, R.S. Somerville, J.R. Primack and A. Dominguez, Semi-analytic modeling of the EBL and consequences for extragalactic gamma-ray spectra, Mon. Not. Roy. Astron. Soc. 422 (2012) 3189 [1104.0671]. 